\title{
FORTY YEARS OF MUTATION RESEARCH: A PILGRIM'S PROGRESS*
}

\author{
CHARLOTTE AUERBACH \\ Institute of Animal Genetics, West Mains Road, Edinburgh EH9 3JN
}

I FEEL honoured by the invitation to deliver the Mendel Lecture. In choosing a topic for it, I have been conscious of the fact that I have been in mutation research for 40 years. I thought that it might be interesting for this audience, many of whom have no research experience going back so far, and many of whom were not yet born when I started research, to hear of the changes in the concepts, problems, techniques, conclusions that have taken place in mutation research during these four decades. I do not intend to be in any way exhaustive, but shall dwell mainly on those changes that have affected my own thinking and work. I also intend to stress my own victions for whatever they are worth. Foremost among these are two:

(a) Mutation is a process that takes place inside living cells, and as such cannot be described fully by a purely physical or chemical model however ingenious.

(b) The advent of the double helix has meant a tremendous breakthrough in our understanding of mutation; but it has neither been the beginning nor the end of mutation research. One gets the impression that molecular geneticists labour under two misapprehensions: (1) that no meaningful questions about mutation could be asked, even less answered, before 1953 and (2) that all questions were answered by the Watson-Crick model. I shall try to dispel these notions.

I had the great good fortune to be recruited to mutation research by H. J. Muller who spent the last year before the second world war in our institute in Edinburgh. This was some years after I had obtained my Ph.D. degree on a problem of development in Drosophila, and I was engaged in following up inherited developmental disturbances. One day Muller came to visit me in the laboratory. He sat down next to me and asked me what I hoped to achieve with my work. I said I wanted to find out something about the action of mutant genes. He replied that there are thousands of genes for which this might be studied, but that none of these studies really led back to the gene itself-which was, of course, true in 1938. "If you want to find out something about the gene", he said, "you have to study how it can be made to change by mutation ". This conversation set me on the path of my pilgrimage in mutation research. I have never regretted that I chose it.

The year that Muller spent in Edinburgh will be unforgettable to his small group of students and research workers. Among the latter were Pontecorvo and Koller. There were regular discussions that ranged far beyond problems of mutation; but for the present purpose only the latter are relevant. 1938/39 were the years when the target theory was at its height.

* The Mendel Lecture, delivered at the 15th Meeting of the Genetical Society, 12th November 1977.

$40 / 2-\mathrm{A}$ 
It had grown out of Muller's discovery, 10 years earlier, that X-rays produce mutations and chromosome breaks. Subsequently, a group of geneticists, foremost among them Muller and Timoféeff-Ressovsky and of physicists, mainly Zimmer and Delbrück, studied the kinetics of these processes on the assumption that genetic effects are produced when chromosomes are " hit" by ionisations, i.e. when an ionisation occurs within a chromosome or extremely close to it. They arrived at two conclusions, which form the basic tenets of the target theory. (I) A mutation is produced when an ion or small ion cluster hits a gene. (2) Ions or ion clusters can also break chromosomes, and if a broken chromosome does not rejoin in the old way-as the majority do-two broken ends may join up in a novel way, forming a rearrangement like an inversion or a translocation.

Thus the first station on my pilgrim's path was a shooting range with bullets being fired at random and occasionally hitting one of the fixed targets. It was an exhilarating and inspiring sight, and indeed the target theory has remained impressive to the present day. The field was surrounded by a fence with meshes fine enough to prevent stray bullets from leaving it and outside disturbances from entering; for the target theory was supposed to cover the whole of mutagenesis including spontaneous mutation. It was this claim to universality which eventually destroyed the monopoly of the target theory in mutation research. Biological phenomena are too complex to be contained by even the finest-meshed wire; they have a way of creeping out into new open ground. Muller, in his review of Schrödinger's book on the physical basis of the target theory, stressed that the final picture of mutagenesis must contain chemical as well as physical forces. Phenomena that could not be fitted into the strictly physical picture had turned up already then; but for the time being we ignored them because there was still so much to explore within the target field.

Let me mention some of the problems that occupied us. One of them was actually being investigated by Muller's students in Edinburgh, and simultaneously by Bauer in Germany. It concerned the origin of chromosome rearrangements. There were two schools of thought in this regard. One, led by the Russian geneticist Serebrovsky, considered that rearrangements were "illegitimate cross-overs" and were formed when two different chromosomes or regions of the same chromosome happened to lie in juxtaposition during irradiation, so that both were broken by the same hit. The other, led by Stadler, assumed that breakage always preceded rearrangement formation and that any two broken ends, provided they were not too far apart from each other, might join up to form a rearrangement. The target theory offered a clear means of deciding between the two models. Serebrovsky's model required only one hit per rearrangement; the frequency of rearrangements should therefore increase linearly with dose. Stadler's model required two independent breaks, and rearrangement frequency should increase as the square of the dose. After the war, when communication between German and Allied scientists became possible again, it turned out that Stadler's breakage-first hypothesis had been confirmed by both groups of workers.

The target theory has remained a guiding principle in mutation research. It is now applied to ultraviolet light and chemical mutagens. If a treatment yields an exponential killing curve and a linear mutation curve, it is assumed to act by single " hits" on the genetic material. If the curves deviate from 
these simple shapes, different models are offered. Let me here insert a word of caution about the interpretation of non-linear dose-effect curves for mutation induction, especially upward-bending ones. In the case of $\mathrm{X}$-ray induced rearrangements which I have just discussed, one of the two models-that of Stadler-demanded a quadratic curve, and when such a curve was obtained, it could be used in evidence for the hypothesis. Where, however, no such a prior model exists, it is very hazardous to conclude that data which can be fitted to a quadratic curve must indicate a two-hit mechanism. Quite often, the same data can be fitted equally well or better by other curves, including bi- or polyphasic ones in which linear stretches of increasing steepness follow each other. Such curves may be obtained when the same treatment that causes genetic lesions alters the environment in which these lesions have to develop, e.g. by inhibiting a repair process.

In one respect, the kinetic analysis of X-ray induced effects proved disappointing. Muller had hoped that it would allow a distinction to be drawn between mutations that inactivate individual genes and small deletions that remove a whole gene or several contiguous ones. This is an extremely important question for the assessment of genetic damage from radiation and chemicals for, while a mutation within a gene need not be harmful, removal of several genes almost always is so. Muller argued that, since deletions are due to the removal of a piece of chromosome, they require two breaks. Their frequency should therefore increase as the square of the dose like that of translocations. In fact, it turned out that this was true only for very large deletions. The small ones, i.e. just those that are so difficult to distinguish from gene mutations, behaved like gene mutations in showing linear induction kinetics.

The origin of these small deletions of one or a few genes is still something of a puzzle since they differ from large chromosome rearrangements in a number of properties. At the time I am speaking of, their linear dose dependence contributed to the beginning doubts regarding the accuracy of the target theory for ionising radiation. Muller argued that if a single hit can delete several contiguous genes, then the energy from an ionisationa " hit"-must be able to spread over a certain distance. Other observations pointed in the same direction. Conditions that are known not to interfere with the primary action of radiation yet were found capable of modifying its effect. Thus, in most systems a given dose of radiation produced more mutations and chromosome breaks in the presence than in the absence of oxygen. Again, the spermatozoa of Drosophila yielded several times as many mutations after a given exposure as did the spermatogonia. A way out of this puzzling situation was shown by radiation chemistry. When viruses or enzymes were inactivated in vitro in solution by ionising radiation, a large part of the radiation energy was found to reach the target not by direct hits but indirectly through water radicals. Oxygen modifies the types, frequencies and life spans of these radicals. Organic molecules compete with the target molecules for radicals and thus act as protective agent. I remember the time when it was a hotly debated question whether in the cells of higher organisms X-rays act on the chromosomes mainly by direct or indirect action and whether differences in sensitivity between cells are wholly due to different degrees of oxygenation and different types and amounts of scavenger molecules. Interest flagged when calculations showed that inclusion of all possible indirect effects in the target would no more than 
double its size. This was not sufficient to account for the observed discrepancies.

But now a new concept arose in mutation studies, that of repair. Already in 1952, Thoday had postulated that radiation-induced chromosome breaks may remain latent for some time and that only some of them will develop into actual breaks. A few years later, the Russells obtained data on mice which they tentatively attributed to a repair process acting on potential gene mutations. They found that, in mouse spermatogonia and oocytes, a given dose of radiation produced several times as many mutations when applied acutely than when administered as chronic treatment. This was in contrast to experiments on Drosophila spermatozoa-and later ones on mouse spermatozoa-in which the frequency of mutations is independent of the rate at which the radiation is applied. The Russells suggested that spermatogonia and oocytes, but not spermatozoa, might possess a repair mechanism which is inhibited by acute radiation. Nowadays, when we know a lot about repair processes in DNA, this hypothesis seems plausible. In the late fifties it created a sensation and was received with incredulity. Not only did it contradict one of the tenets of the target theory-the doserate independence of radiation damage. It also had a very important bearing on the estimates of genetic damage from fall-out and nuclear waste. If human spermatogonia should behave like those of the mouse, then chronic exposure to low radiation doses in the environment should be much less damaging than had been extrapolated from experiments with acute exposure. Indeed, some newspapers seized upon the Russells' findings in order to play down or totally dismiss the genetic risks from radiation. I tried hard, but in vain, to get an answer to one of these ill-informed articles into the Manchester Guardian. The assessments of radiation hazards which are produced regularly by the United Nations and other associations take account of the correction made necessary by the Russells' data. For chemical mutagens, comparisons between acute and chronic treatments with, for example, suspect drugs is extremely important but so far has hardly been studied.

To return for a few minutes to the immediate pre-war year in Edinburgh and the problems that occupied us then. Many of them turned upon the nature of the two main effects of X-rays: gene mutations and chromosome breaks. Were these essentially different from each other? In particular, did X-rays produce true gene mutations or were these in reality all due to small deficiencies or position effects of neighbouring rearrangements? This last question led to vehement arguments between two schools of thought. One, led by Muller, claimed that X-rays can produce true mutations; the other, led by Stadler (who had discovered the mutagenic action of X-rays in maize) claimed that they cannot. Both schools had experimental evidence for their claims; but both had also arguments for doubting the evidence of the other. This was one of the problems that finally was solved only in the era of molecular genetics, when it could be shown that X-rays can produce base changes in DNA, thus confirming Muller's claim. On the other hand, Stadler's finding that all X-ray induced mutations at a particular locus in maize were small deficiencies has recently been confirmed for another locus. Probably, the discrepancy is due to the nature of the organisms with which the experiments were carried out: Drosophila and maize. Plant chromosomes are broken by doses that are far below those required for the same 
effect in Drosophila. This, incidentally, was one of the little biological facts that could not be fitted into the strict target theory.

I want to forestall the criticism that we were spending much effort on questions that, after all, were too specialised to be of general interest. I want to make it clear that, taken in its full context, our quest was anything but specialised. On the contrary, it dealt with a problem that is still under study: the structure of the chromosome. The model in vogue at the time was that of " beads on a string ", i.e. of genes separated from each other by intergenic linkers. This model was then under strong attack by Richard Goldschmidt. In his opinion, development was controlled by integrated chromosome sections or even whole chromosomes. Mutations were not changes of individual genes, but rearrangements within or between chromosomal regions. He had some support from the existence of position effects but, apart from the fact that position effect is not a general phenomenon, there is a logical difficulty in this view. If all mutations are due to rearrangements between chromosomal sections, then these sections must already differ from each other, and at least these original differences must be attributed to genuine mutations. Nevertheless, Goldschmidt carried his claim to the point where he could write: "The gene is dead". I remember how much this upset Muller. On the whole, the balance of evidence at that time pointed to a separation of genes by non-genic linkers; but final proof was not possible with the means then available.

Concern with the structure of the chromosome naturally led to the question of the limits of the gene. Both Stadler and Muller pointed out that these limits may be defined in three different ways depending on whether one considers the gene as unit of mutation, unit of recombination, or unit of function (i.e. non-complementation). We are now more familiar with these units as Benzer's muton, recon, and cistron.

As regards gene action, no enzyme studies were as yet available. But very ingenious techniques of manipulating the numbers of mutant and wildtype alleles in Drosophila led Muller to a classification of mutant genes that subsequently has been given a biochemical basis by research on mutant enzymes. Thus, Muller's "amorphs", now usually called null-alleles, yield no enzyme or cross-reacting material. His "hypomorphs" do the same as the normal allele but do it less efficiently because they produce either a less efficient enzyme or less of the normal one. His " antimorphs", which antagonise the action of the normal allele, in several cases have been shown to do so by forming polypeptides which, in a multimeric enzyme, interfere with the action of the normal polypeptide chain or chains.

Underlying these various problems was the basic one of mutation research and, indeed, of genetics in general: what enables a mutated gene to replicate at once as faithfully and persistently in its new form as it did previously in its original one? Two main types of model were discussed. One was based on specific attractions by resonance forces; the other on an antigen-antibody type of replication. The concept of complementarity as an immediate means of achieving identical replication was first put forward in our institute by Dr Kacser and Dr Oftedal. They pictured the gene as a double structure between a strand of nucleic acid and a strand of protein, the two being held together at the interface by specific surface forces. At replication, the same forces made it possible for the nucleic acid moiety to attach to itself a complementary protein moiety and vice versa. It was 
a model that impressed us by its ingenuity, but as it was published only several years later, when the Watson-Crick model was already generally accepted, it had no future.

The double-helix model, as you know, finally and unequivocally solved the basic problem of mutation. In my experience, it is very rare to get a complete and final answer to a biological question; when it does happen, it is extremely satisfactory. Yet, as I mentioned at the beginning, the double helix left many mutation phenomena unaccounted for. Some of them had already accumulated during the reign of the target theory. More of them came to light on two new paths that were opened up to mutation research round about 1940. One was chemical mutagenesis, the other was mutation research on micro-organisms, from viruses to fungi. Curiously enough, these two paths remained almost completely separate for several years: chemical mutagens were analysed in Drosophila, while micro-organisms were treated almost exclusively with ultraviolet light. Yet, when the two paths converged and fused at the end, they were found to have led to the same revolutionary concept: that of premutational stages in DNA which may or may not develop into gene mutations or chromosome breaks.

Up to then, my own path had been that of a modest observer, taking part in discussions on radiation genetics, and testing-unsuccessfully-a few carcinogens for mutagenic activity in Drosophila. Now, however, I joined the road of chemical mutagenesis. This road had started many years before in the early days of genetics as a tiny and uncertain track, leading to no clear conclusions and soon abandoned. At the beginning of the Second World War, two Edinburgh pharmacologists, Prof. A. J. Clark and Dr J. M. Robson, investigated the properties of mustard gas in view of the possibility that it might again be used by the Germans. Like the pharmacologists in the First World War, they were struck by the similarities of the effects of mustard gas and those of X-rays. Both agents produce wounds that heal only with difficulty and that, once healed, have the tendency to break open again. As late as 1939, ophthalmologists were treating ulcers of the cornea that had been produced by mustard gas in the first war. In the intervening years, it had become clear that the persisting effects of $\mathrm{X}$-rays are at least in large part due to chromosomal damage. It seemed possible that this applied also to mustard gas. Before asking me to test this hypothesis on Drosophila, Dr Robson obtained a result that strongly supported it. He applied hormonal stimulation to the vaginal epithelium of rats that had or had not been previously exposed to mustard gas. In the untreated ones, hormone treatment caused a burst of mitoses; in the treated ones, it did not. Thus mustard gas, like X-radiation, inhibits mitosis. The question was whether, like X-rays, it does so by producing lesions in the chromosomes and, if so, whether these lesions can lead to mutations.

So I started experiments with Drosophila. This stretch of my pilgrim's progress was the stoniest and most demanding one, but also the most thrilling. New vistas opened at every step. The apparatus we used at first was very primitive, and all of us-Robson, myself, and several young people who helped with the exposures--got burns and rashes. For many weeks I had both hands bandaged. The dermatologist warned me never to go near mustard gas again because of the risk of worse consequences: the antigenic properties of mustard gas had just been discovered. By that time, however, the results had already become so exciting that we could not 
possibly stop the work; we only became more careful and developed safer exposure techniques. When preliminary tests had confirmed that mustard gas inhibits mitosis also in Drosophila, I did the first mutation experiment. Its result far exceeded our expectation. While all previous experiments with chemical substances had at best given barely significant increases over the spontaneous mutation frequency, mustard gas had yielded a number of mutations that would have been obtained by irradiation with 2000 or $3000 \mathrm{R}$ units. Soon we established that mustard gas produces the whole range of mutations and chromosome rearrangements that are found after X-ray treatment: dominant and recessive lethals, dominant and recessive visible mutations, chromosome breaks and all known types of chromosome rearrangement. Compounds that were related to mustard gas-some but not all of them war gases-had similar effects. Because of the nature of these chemicals, all our results were "classified"; the first short note on them appeared in "Nature" in 1946 and the first full papers came out in 1947. Some of my colleagues thought that this must be very frustrating for me. On the contrary, it suited me well. Under protection of the secrecy act, I could follow up my ideas without pressure for publication and without fear of competition.

After the war I learned that other, less strongly and universally acting mutagens, foremost among them urethane, had meanwhile been discovered by Oehlkers in Germany. In Russia, Rapoport found that formaldehyde mixed with the food of Drosophila larvae is an extremely efficient mutagen. When his research was stopped by the total eclipse of genetics under Lysenko in Russia, several workers in the West, including myself and a few of my students and co-workers, analysed the very interesting effects of formaldehyde. Rapoport also discovered a whole range of new mutagenic compounds, e.g. epoxides; but these results reached the West only after a long delay, when the mutagenic ability of most of these compounds had been re-discovered independently by Western geneticists. From the 1950's up to the present time, the number of chemical mutagens has kept on increasing. It seems as though the first discoveries had removed a psychological barrier against tests of chemicals, set up by the contrast of the early failures in this area and the triumphant success of radiation mutagenesis.

I personally did not feel tempted to test large arrays of chemicals for mutagenic ability. I knew that my chemical knowledge was quite inadequate to the task of forming my own working hypotheses in this field. I should have to test the hypotheses of chemists, and where would be the fun of this? Moreover, unlike some of my colleagues, I did not believe that knowing which chemicals are mutagenic and which not would yield information on the chemical nature of the gene. This had not happened in the case of carcinogenesis. It was not likely to happen in the case of mutagenesis. Indeed, it did not happen. On the contrary, it was knowledge of the nature of the gene, acquired by non-genetical methods, which led to insight into the action of chemical mutagens and to the discovery of wholly new classes of them, like the base analogues and acridines.

My own interest concerned the process of mutation. I thought that a comparison between the effects of X-rays and of chemical mutagens might throw light on it. I have mentioned already that the similarity of effects was striking; but this did not impress me particularly. After all, with the methods then available we could detect only two endpoints, mutation and 
chromosome breakage, and these were not necessarily produced in the same ways by different treatments. I was much more interested in the differences between the effects of X-rays and chemicals. These, I thought, might reveal steps in the mutation process that did not occur or were not easily detected in radiation mutagenesis.

This turned out to be true. One difference concerned the sensitivity pattern of the testis to radiation and chemicals. Some chemicals resemble $\mathrm{X}$-rays in producing more mutations in post-meiotic than in pre-meiotic stages; a few do the opposite. Still others affect preferentially meiotic and peri-meiotic stages. The most specific mutagen in this respect is formaldehyde-treated Drosophila medium, which acts exclusively on the early larval spermatocytes. In spermatogonia, in which it fails to produce mutations, it still induces crossing-over. The importance of this observation lies in the lack of correlation between mutagenic and recombinogenic effects of a treatment. This needs to be taken into consideration nowadays, when the testing of suspected mutagens often has to rely on the correlation between mutagenic ability and more easily observed effects like recombination, which in themselves do not constitute a genetic hazard. It also has a bearing on the problem, well known from bacterial genetics, to what extent the pathways leading to mutation and recombination are common or interconnected.

Some of the early experiments with chemical mutagens gave results that were of interest for the old problem of the relation between gene mutation and chromosome breakage. In general, the ratio between rearrangements and mutations is lower for chemicals than for X-rays; but also in this respect chemicals differ greatly from each other. These observations lent support to the model of a chromosome consisting of genes in which mutations are produced, and intergenic linkers that are more or less easily broken by different agents. Proof that chromosome breakage differs essentially from mutation was obtained when it was found that, in Drosophila spermatozoa, alkylating agents produce two types of primary lesion, which can be distinguished by their response to prolonged storing in the seminal receptacles of untreated females. One type, leading to breakage, increases in frequency during storage; the other, leading to mutation, does not.

Both the storage effect and the great variation of the ratio between rearrangements and gene mutations after chemical treatment supported the "beads on a string" model, but they did not prove it. This has been achieved only recently when molecular and electron-microscopic analysis revealed the existence of "linkers" or "spacers" between genes. Indeed, the " beads on a string " model has been shown to apply to dimensions smaller than a gene: an observation that still awaits full explanation.

I have left to the last the two most important peculiarities of chemical mutagens: their tendency to produce pre-breaks and pre-mutations. By pre-break I mean a damaged site in a chromosome which may or may not result in chromosome breakage. By pre-mutation I mean damage to a gene which may or may not result in a fixed, i.e. replicated, alteration. The storage effect in Drosophila and a similar one in plant seeds had shown the existence of pre-breakage lesions. That of premutational lesions was inferred from experiments on Drosophila and, later, on micro-organisms where chemically induced mutations often appear after a delay of one or more cell cycles. In many cases, a premutated gene may give rise to the same mutation for several or many cell generations. This has been most 
clearly documented in fission yeast, where successive mutations could be allocated not only to the same gene-as in Drosophila-but also the same or a closely neighbouring site within this gene. The most remarkable feature about these instabilities, however, is their ability to replicate in the unstable state, one unstable gene giving rise to two or more of its kind. The explanation of these chemically induced " replicating instabilities" still eludes us and is not necessarily, nor even probably, the same in all cases. Sometimes duplications may create instabilities by oblique crossing-over between or within genes. At the moment, it may be best to bracket the chemicallyinduced instabilities with those of spontaneous origin, many of which are due to movements of controlling elements or insertion sequences.

Whatever the final explanation of chemically-induced delayed mutations and instabilities, they pointed to the existence of pre-mutated states of treated genes. Meanwhile, research on $U V$-induced mutations in bacteria had led to the concept of pre-mutations by evidence of a very different nature. The crucial discovery was that the mutagenic effect of a given dose of UV can be profoundly modified by post-treatment conditions. Thus, either exposure to short-wave visible light after irradiation or absence of amino acids in the plating medium were found to reduce mutation frequencies drastically. Tentatively, both phenomena were attributed to "repair" processes, a hypothesis which implies the existence of a pre-mutated state. While molecular studies of a much later period have fully vindicated this explanation, alternative ones could not be excluded in the late forties and early fifties when these phenomena were discovered. An assumption that linked the previously separate paths of chemical mutagenesis in Drosophila and UV-mutagenesis in micro-organisms considered that UV acts via the production of a mutagenic chemical, which is destroyed by visible light and decays in the absence of amino acids. This hypothesis seemed to gain support from the finding that mutations are produced in bacteria that grow for some time in UV-irradiated medium. It is true that, in these experiments, the energy and wave lengths required for mutagenic effect differed from those required for mutagenesis by direct irradiation; but it was not difficult to explain these discrepancies by secondary assumptions. We now know that UV-irradiation of bacterial medium does indeed produce mutagenic peroxides; but we also know that direct irradiation acts in a completely different way.

Thus, when the path of UV-induced mutagenesis in micro-organisms had joined that of chemical mutagenesis in Drosophila, it turned out that both had arrived independently at the same concept, that of pre-mutational and pre-breakage states. Now this concept had been expressly excluded in the premises of the target theory. The kinetic analysis of X-ray-induced mutagenesis and rearrangement formation had been undertaken only when preliminary tests had established that irradiation produced mutations and chromosome breakage immediately in the damaged genes or intergenic linkers. There had, in fact, been indications of a pre-breakage state even in $\mathrm{X}$-ray experiments; but this was just one more of the slightly disquieting findings which had crept in through the fence round the target theory without seriously damaging it. Now, however, we felt that a really big gash had been made in this fence and that completely new avenues had opened up for mutation research. While we were exhilarated by this prospect, we also felt frustrated by lack of an alternative framework that 
could accommodate both the old and the new findings. What we needed most of all, was knowledge of the chemical nature of the gene and of its mode of replication. This, as you know, was provided in the years following 1953, when the double-helix model came to be generally accepted.

The new discipline of molecular genetics developed with dazzling brilliance and rapidity. For mutation research, the gain was tremendous. Not only did we now understand why mutated genes replicate as such. We also knew what kinds of change in DNA can produce mutations and, for a number of mutagens, we knew or at least had testable hypotheses by what kind of change they do produce mutations. We realised that, in principle, this knowledge would be obtainable for any present or future mutagen. For many of my colleagues, especially those coming from physics and chemistry, mutation research seemed to have reached its final goal, with no essential problem left unsolved.

When I had made my way to the new summit of mutation research, I found myself in a studio that was full of beautiful clean models of chemical compounds-purines, pyrimidines, nucleosides, nucleotides, acridines, base analogues, alkylating molecules-all surrounding a huge model of a double helix. I was shown how the mutagenic molecules could be fitted to the helix, and how this affected the bonds that held the structure together and the attractions that led to replication. It was extremely impressive. And yet I felt dismayed; for the outside view was excluded even more effectively by the walls of this studio than it had been by the meshes of the fence round the target theory. Coming in from the tangled undergrowth of biological phenomena, with its thorns and vines sticking all over me, I felt like an intruder in this austerely clean environment. What seemed to have happened -and what indeed did happen for the next 8 or 10 years-was that the rigidly physical view of mutagenesis had been replaced by a rigidly chemical one. All mutational phenomena were explained in terms of reactions between DNA, its precursor molecules and mutagens. Spontaneous mutations were due to tautomeric shifts in the nucleic acid bases. Alkylating agents acted by depurination or ionisation. Mutagen specificity was due to the reaction of chemicals with specific bases. Delayed mutations occurred when an apurinic site or an alkylated guanine acted repeatedly as template for replication, etc. In fact, in 1960, Lederberg could say that the bacterium was far too complex a genetic entity for experiments on mutagenesis, and that the best system for such a study was DNA in a test tube.

I felt sure that this could not be the whole answer. We had, of course, always known that the mutation process must start with a change in a gene whatever its chemical nature. It was a great step forward that we knew what these chemical changes were. But I could not believe that this solved the whole problem of mutagenesis. It was inconceivable to me-and must have been inconceivable to many biologists - that mutagenesis should differ from all other living processes in not being directed by the complex machinery of life, its enzymes, its interacting biochemical pathways, its membranes, its regulatory abilities. Moreover, I knew of many observations that could not be accounted for by simple chemical reactions of DNA, and more of them constantly came to light, such as mutator genes, "hot spots", mutagen specificities, replicating instabilities.

For quite a few years, the hegemony of nucleic acid chemistry in mutation research was so strong that it was difficult to take a stand against it. I 
felt like a missionary preaching to the unconvertible except that, unlike a missionary, I was preaching doubt and attacking beliefs. What in the end made breaches in the wall of the nucleic acid studio were, however, not my sermons but some of the stubborn facts of nature that had previously demolished the fence around the target theory.

Foremost among these were two: mutator genes and dark repair. Mutator genes showed the involvement of enzymes in DNA-replication. Dark repair uncovered several complex enzyme systems, some of which remove pre-mutations while others fix them. With the introduction of enzymes into mutation research, the step was made from a study of the simple chemical reactions of DNA to biochemistry in the living cell, where enzyme specificities and interactions, genetic background, metabolic conditions etc. are expected to play important roles. Previously inexplicable phenomena, such as modifications of mutation frequencies by genetic background, differences between the mutational responses of the same gene in different genotypes, or between sites within the same gene, could now be attributed, at least in principle, to, e.g. effects on enzymes involved in replication or repair, competition between repair enzymes for recognition sites in DNA etc. At the moment, dark repair seems to be the most rewarding object of study. Indeed, it threatens to take over the monopoly previously exercised by nucleic acid chemistry. There is a tendency to attribute every unexplained observation to differences in repair enzymes. Often this may be the correct explanation; but we should not forget that the mutation process includes steps beyond repair: transcription, translation, formation of a new enzyme or loss of an existing one, changes in ribosomes, alterations in membrane structures etc., all of which can be subsumed under the term "expression".

It is true that the expression steps in mutagenesis are likely to differ between groups of genes, e.g. forward and reverse mutations, and even between genes involved in different biochemical pathways, e.g. reversions of arginine and methionine auxotrophs. It might therefore seem that a study of these steps will not add much to our understanding of the general principles of mutagenesis. This may be true; but for me the most exciting prospect in present-day mutation research is that it seems on the point of linking up with other branches of research on cell biology. Already we know that enzymes required for certain types of error-prone dark repair are also involved in other processes such as recombination and phage induction. Analysis of the conditions for the expression of a new mutation to prototrophy may be one of the means for studying the concatenation of biochemical pathways in the cell. Altogether, I feel that mutation research has reached a stage when it can look ahead to whole new areas of exploration.

Let me finish by telling you a little story about what happened in Edinburgh not so very long ago. At the official opening of the Institute of Molecular Biology, Jaques Monod, as one of the fathers of this discipline, gave a very stimulating talk. At the end of it he pointed out that molecular biology appeared to have already reached and even passed its peak. He was looking forward to an era of research into what he called " metabiology". With this he meant a study of the ways in which molecular processes are translated into the structural, physiological and biochemical features of the living cell and organism. At present we are already in the midst of this era. Where I disagree with Monod is that I should not call it the beginning of metabiology but the return to biology. 